\title{
Displacement and Dilaceration of Permanent Teeth as a sequel of trauma to Primary Teeth diagnosed bu CBCT: A Rare Cose Report
}

\author{
Dr Pooja Pokharel,' Dr Shruti B Nimbeni,2 Dr Basavaraj S Nimbeni, ${ }^{3}$ Dr Sourav K Rout4 \\ 'Dept of Orthodontics, ${ }^{2,3}$ Dept of Pedodontics, ${ }^{4}$ Dept of Oral Surgery \\ Chitwan Medical College, Bhartapur, Nepal
}

Correspondence: Dr Pooja Pokharel; Email: koiralapooja@gmail.com

\section{ABSTRACT}

Dentoalveolar trauma to the primary teeth can result in displacement, dilaceration, discoloration, delayed eruption and impaction of permanent teeth. Displacement and dilaceration of permanent teeth is the most common sequel of such injuries. The severity of such condition depends on the force of impact, region of trauma and stage of tooth development of the permanent tooth.

In the present case, there was dentoalveolar trauma to primary teeth as a result of which there was displacement of the permanent incisors into the nasal floor and 90 degree dilaceration at the cementoenamel junction. The treatment included surgical removal of the displaced teeth, exposure of palatally impacted canines followed by orthodontic correction and prosthodontic replacement of the extracted teeth.

Cases of dentoalveolar trauma should be critically evaluated and advanced diagnostic aids like CBCT must be used for a clear picture of the case and better treatment planning. Treatment of such cases should be followed up for a longer period of time.

Keywords: Cone Beam Computed Tomography, dilaceration, displacement, palatal impaction of canine

\section{INTRODUCTION}

Dental trauma has emerged as the most prevalent dental diseases after dental caries.1.2 Trauma to the permanent incisors are due to direct injury to the incisors or secondary to trauma to primary teeth resulting in various developmental defects. ${ }^{3}$ The prevalence of dentoalveolar trauma in primary teeth is $11-13 \%$ but the incidence of dilacerations and displacement of permanent teeth due to such trauma is rare. ${ }^{4}$ Diagnostic aids like CBCT helps in appropriate treatment planning with multidisciplinary approach. The present case reports the displacement and dilacerations of permanent maxillary incisors due to dentoalveolar trauma to primary dentition diagnosed by CBCT.

\section{Case History}

A thirteen-year-young girl patient reported to the department of Pedodontics and Preventive Dentistry with

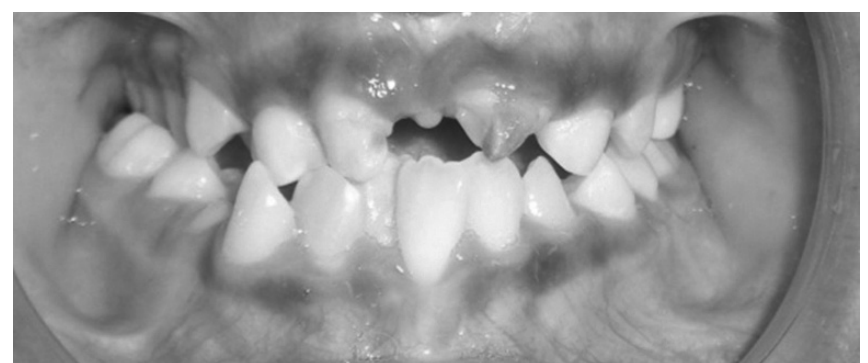

Figure 1: Intraoral Frontal View a chief complaint of delayed eruption of teeth in the upper front region of the mouth.

The child was healthy and there was no history of systemic illness until she was one and half years old. According to her father, there was a history of craniofacial trauma due to fall from the fourth floor twelve years back when she was one and half year old. The primary central incisors that had erupted were avulsed due to trauma. The patient was treated in Intensive Care Unit for 2 days. She stayed in the hospital for one and half month and was later discharged.

Extra oral examination showed a straight profile. Intra oral examination showed anterior cross bite, missing \#11, 21, hypoplastic \#12, 22 and crowding was observed with mandibular incisors. There was class I molar relationship with posterior cross bite on the right side (Figure 1). The maxillary arch was narrow and constricted. Radiographic examination was done by lateral cephalograph and orthopantomograph which showed impacted \#11, 21 and hypoplastic \# 12, 22 (Figure 2).

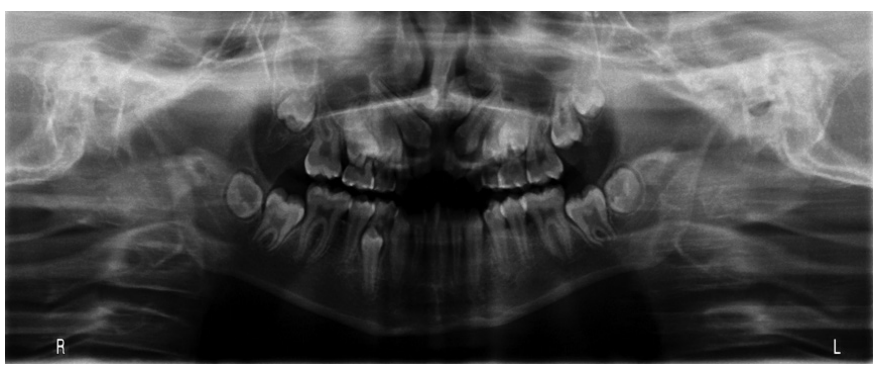

Figure 2: Orthopantomograph showing displaced \#11, \#21 


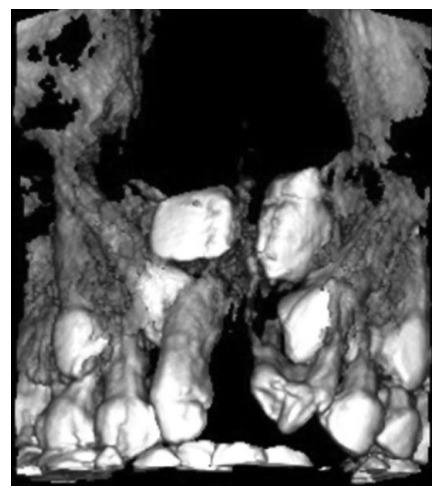

Figure 3: $\mathrm{CBCT}$ Frontal View displaced \#11, \#21

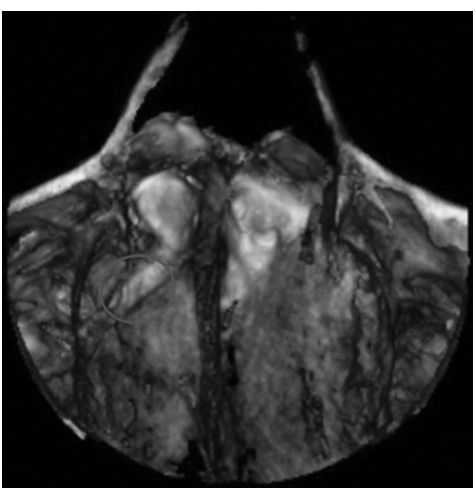

Figure 4: $C B C T$ Vertex view showing Odontome distal to \#21
Further investigation was done by Cone Beam Computed Tomography (Planmeca ProMax 3D) of the maxilla. Dataset scan at intermediate resolution $0.4 \mathrm{~mm}$ with Planmeca Romexis software was used. It was seen that the $\# 11,21$ was impacted in the nasal floor and dilacerated at the CEJ. \#22 was found to be rotated mesio lingually (Figure 3). Palatal impaction of the permanent canines was also observed. The presence of an impacted calcified structure, palatal to the crown of \#21 was observedbut it was not well distinguished (Figure 4).

Treatment of the present case was done in phases. In the first phase bonding of the premolars \# 14,15 and 24 was done with Enlight light cure adhesive (ORMCO, CA, USA) and banding of \#16, 26 was done. In the second phase, surgical extraction of \#11,21 and was performed under general anesthesia as the procedure was invasive and there were chances of profuse bleeding due to the close proximity of the impacted teeth to the nasal floor. During the extraction impacted calcified structure palatal to the root of \#21 was also extracted.Surgical exposure of the permanent canines was done with full thickness flap using closed eruption technique and bonding of the permanent canines with lingual buttons in order to facilitate canine movement. Ligature wire was engaged from the lingual buttons to the brackets on the premolars (Figure 5). Therapeutic extraction of \#63 was also done during palatal flap reflection. In the third phase, extraction of the \#53 and the hypoplastic \#22 is planned under local anaesthesia at a later date keeping in mind that there was less number of teeth in the anterior region already. In the final phase, alignment and levelling of the permanent canines is planned with MBT system.

\section{DISCUSSION}

Fall injury is found to be the most common cause of traumatic injury to primary dentition. The most susceptible age for these traumatic injuries is 2-4 years, when the physical activity of children increases, as they learn to explore their surroundings. 3 Maxillary anterior teeth are the

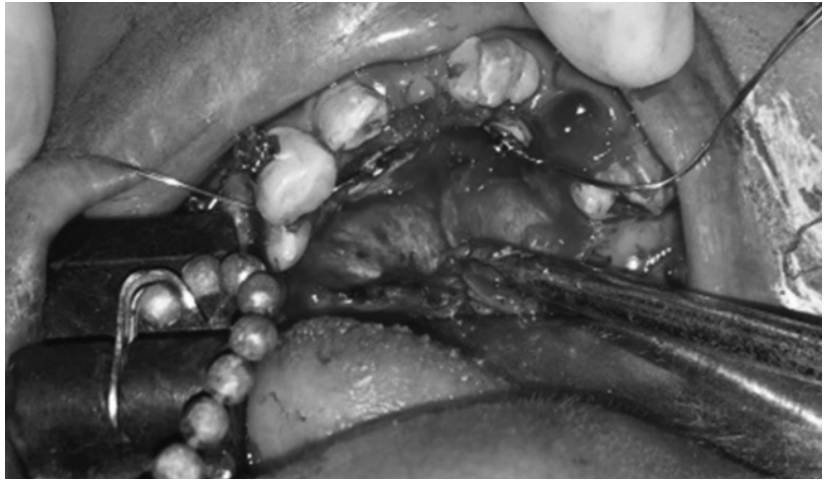

Figure 5: Surgical exposure of Palatally impacted canines

most frequently affected teeth due such trauma. ${ }^{5}$ Such traumas usually result in axial impact, which dislocates the teeth into the tooth socket leading to dilaceration and dislocation and impaction of permanent teeth as found in our case. ${ }^{6}$ The term "dilaceration" is applied to the teeth that have the long axis of either the whole or part of the root formed at an angle to the crown. Such anomalies may be as a result of trauma to the primary tooth during the development of the permanent tooth. ${ }^{3}$ Variations in the degree of dilaceration and impaction depends on the age during the occurrence of injury and the direction and severity of the force transmitted from the affected primary tooth to the unerupted permanent tooth germ and the stage of development of the permanent tooth.?

In the present case, patient sustained the injury due to fall from the 4th floor when she was one and half year old.At this time, crowns of \#11, 21 were still forming. Her primary teeth got avulsed and the force of impact resulted in displacement of the developing tooth germ of \#11,21 into the nasal floor. Further as the development of the displaced teeth progressed, there was dilaceration at the cemento- enamel junction and the root was bent in 90-degree angulation in the nasal floor. \#12 was also affected leading to hypoplasia. \#22 was hypoplastic and crown was rotated.

In cases of dento alveolar trauma diagnosis and the diagnostic aids used are very crucial for identifying the exact location of the traumatized and displaced tooth and to aid in better surgical planning.

For the present case, an orthopantomogram was taken which showed impacted and dislocated crown of 11,21. The exact morphology of the impacted tooth was not clear so further evaluation was performed by CBCT.

CBCT is advantageous compared to conventional radiographs. It analyses the morphology of impacted tooth in three dimensions. ${ }^{7}$ Most of the technical limitations of the plain film projection were overcome by CBCT which 
provides a 3-D representation of the maxillofacial tissues with less radiation exposure. ${ }^{8}$

CBCT images were provided in the bone window showing Axial, Coronal and Sagittal planes. СBCT findings showed impacted \#11, 21 with the incisal edge present at the apical aspect of \#12, 22 respectively. There was the presence of $90^{\circ}$ dilaceration of roots of \#11, 21 at the level of cemento-enamel junction. The dilacerated portion of the teeth was present labio-palatally and at the nasal floor. There was also the presence of an impacted calcified structure palatal to the root of \#21 but it was not well distinguished. Rotation with \#22 was also visible. Palatal impaction of the permanent canines was also observed which had still not erupted.

The main treatment objective of this case was to extract the impacted incisors followed by orthodontic treatment for the correction of the malocclusion.

The treatment of the present case was done in 4 phases as the patient was undergoing pubertal growth.In the first phase bonding of the premolars \# 14,15 and 24, banding of \#16, 26 was done.

In the second phase, surgical extraction of impacted \# 11 , 21 were performed under general anaesthesia. It was seen that extracted teeth had undergone complete crown formation with small roots which had a $90^{\circ}$ dilaceration. Distal to the root of \#21 another calcified tooth like structure measuring $1 \mathrm{~cm} \times 0.3 \mathrm{~cm}$ that observed in CBCT was also removed and sent for histopathology.

In several cases of palatal canine impaction, early uncovering of the canine during the mixed dentition phase, before the start of orthodontic treatment has been advocated so as to allow the autonomous eruption of canine which reduce the treatment time and helps achieve superior esthetic results..$^{910}$ In the present case, exposure of impacted canine was performed along with extraction of the impacted incisors.

Palatal canine impaction can be treated by 2 methods. Open eruption technique or preorthodontic exposure, where a full thickness mucoperiosteal flap is raised in the area of the palatally impacted canine, the bone covering the lingual surface of the canine is removed and a bracket is bonded over the exposed crown surface of the canine. The flap is repositioned over the exposed crown of the canine with a small window in the flap where the bracket can be accessed for traction. A dressing is given which mechanically attaches to the bracket. The tooth is allowed to erupt autonomously. While in closed mouth technique a full thickness mucoperiosteal flap is raised, sufficient bone covering the crown is removed and a piece of gold chain is attached to the exposed surface of the crown. The flap is repositioned and the gold chain is left to exit through the palatal mucosa. After healing of the mucosa is achieved the tooth is orthodontically guided into the oral cavity. ${ }^{10}$

In cases of palatal canine impaction both open and closed eruption techniques are considered to be appropriate because palatal gingiva is an attached gingiva and any surgical orthodontic treatment should promote natural eruption through the attached gingiva. ${ }^{11}$

In the present case, surgical exposure of the permanent canines was done by raising a full thickness mucoperiosteal flap. Bonding of the permanent canines with lingual buttons was done in order to facilitate canine movement. Ligature wire was engaged from the lingual buttons to the brackets on the premolars. Therapeutic extraction of \#63 was done.

In the third phase, extraction of the \#53 and the hypoplastic \#22 was planned under local anaesthesia at a later date keeping in mind that there was a lack of adequate number of teeth in the anterior region already.

The impact of trauma can be strong enough to dislocate the root of an incisor during avulsion leading to change in the position of the permanent tooth germ which can result in formation of odontome. ${ }^{12}$ In the present case, a calcified structure was visible in $\mathrm{CBCT}$ examination distal to the root of 21 . On surgical removal it was found to be a calcified structure measuring $1.25 \mathrm{~cm}$ in length resembling an odontome. The extracted incisors had undergone complete crown formation with short roots. \# 11 measured $1.5 \mathrm{~cm}$ while \#21 measured $1 \mathrm{~cm}$.

In the final phase, alignment and leveling of the permanent canines was planned with MBT system. The extracted incisors were planned to be replaced by removable partial denture and with implants on a later date. Restorative treatment of enamel hypoplasia for \# 12 was planned using hybrid composite resin to improve the aesthetics of \#12.

\section{CONCLUSION}

Trauma to the primary teeth is mostly neglected by parents and care takers. Early and timely intervention can save patients from undergoing the consequences of such trauma. Clinicians treating such cases must use advance diagnostic aids like CBCT for treatment planning. Growth of the patient must also be taken into consideration so that treatment simulates natural growth and development. Regular and long term follow up of such cases can minimize the sequel of the trauma on the permanent dentition and improve the oral health related quality of life. 


\section{REFERENCES}

1. Irigoyen ME, Mejía-González A, Zepeda-Zepeda MA, Betancourt-Linares A, Lezana-Fernández MÁ, Álvarez-Lucas CH. Dental caries in Mexican schoolchildren: A comparison of 1988-1989 and 1998-2001 surveys. Med Oral Patol Oral Cir Bucal. 2012;17 (5): 825-32.

2. Rawan Fawwaz El Karmi, Mahmoud Anwar Hamdan, Lamis Darwish Rajab, Suha Bassam Abu-Ghazaleh. Prevalence of traumatic dental injuries and associated factors among preschool children in Amman, Jordan. Dental Traumatology 2015; 31 (6):487-492.

3. Andreasen JO, Andreasen FM, Andersson L. Textbook and color atlas of traumatic injuries to the teeth, 4th edn. Oxford: Blackwell Munksgaard; 2007

4. Hamid Jafarzadeh, Paul V. Abbott. Dilaceration: Review of an Endodontic Challenge. J Endod 2007;33(9):1025-1030

5. Volkan Arikana, Saziye Saria, Hayriye Sonmeza. The Prevalence and Treatment Outcomes of Primary Tooth Injuries. European journal of dentistry. 2010; 4:447-453.

6. Diab M, elBadrawy HE. Intrusion injuries of primary incisors.Part I: review and management. Quintessence Int 2000; 31:327-34.

7. Mahesh R, Kani Mozhii G, Sivakumar M. Dilaceration and Eruption Disturbances in Permanent Teeth: A Sequelae of Trauma to Their Predecessors-Diagnosis and Treatment Using Cone Beam CT. Journal of Clinical and Diagnostic Research. 2014; 8(5):10-12

8. Scarfe WC. Imaging of maxillofacial trauma: evolutions and emerging revolutions. Oral Surg Oral Med Oral Pathol Oral RadiolEndod 2005; $100(2): 75-96$.

9. Schmidt A. Periodontal reaction to early uncovering, autonomous eruption, and orthodontic alignment of palatally impacted maxillary canines. Seattle: University of Washington; 2004.

10. Vincent G. Kokich. Preorthodontic Uncovering and Autonomous Eruption of Palatally Impacted Maxillary Canines. Semin Orthod 2010; 16:205-211

11. Dalia Smailiene, Aiste Kavaliauskiene, Ingrida Pacauskiene, Egle Zasciurinskiene, Krister Bjerklin. The European Journal of Orthodontics 2013; 35(6):803-810.

12. Nelson-Filho P, Silva RA, Faria G, Freitas AC. Odontoma-like malformation in a permanent maxillary central incisor subsequent to trauma to the incisor predecessor. Dent Traumatol. 2005; 21:309-312. 